\title{
Técnicas de restauração diretas de dentes anteriores fraturados em paciente odontopediátrico
}

\author{
- Danielle Liêda Cunha Fróes Departamento de Odontologia Restauradora, Universidade Estadual de Londrina \\ (UEL), Londrina, PR, Brasil • Débora Maria Pelisson Lourenço Departamento de Odontologia Restauradora, \\ Universidade Estadual de Londrina (UEL), Londrina, PR, Brasil • Carolina Alves Andrade Departamento de \\ Odontologia Restauradora, Universidade Estadual de Londrina (UEL), Londrina, PR, Brasil • Flávia Barroso \\ Castelani Departamento de Odontologia Restauradora, Universidade Estadual de Londrina (UEL), Londrina, PR, \\ Brasil • Helouise Abreu Laffayett Departamento de Odontologia Restauradora, Universidade Estadual de Londrina \\ (UEL), Londrina, PR, Brasil • Lucas Fernando de Oliveira Tomaz Ferraresso Departamento de Odontologia \\ Restauradora, Universidade Estadual de Londrina (UEL), Londrina, PR, Brasil • João Felipe Besegato Departamento \\ de Odontologia Restauradora, Faculdade de Odontologia de Araraquara, Universidade Estadual Paulista (Unesp), \\ Araraquara, SP, Brasil - Sueli de Almeida Cardoso Departamento de Odontologia Restauradora, Universidade \\ Estadual de Londrina (UEL), Londrina, PR, Brasil - Márcio Grama Hoeppner Departamento de Odontologia \\ Restauradora, Universidade Estadual de Londrina (UEL), Londrina, PR, Brasil
}

RESUMO || O traumatismo dentário (TD) pode comprometer os dentes, o periodonto de suporte e/ou os tecidos moles adjacentes. Frequente em crianças e adolescentes, o TD é um problema de saúde pública mundial, pois, com alta prevalência sobre os incisivos centrais superiores, pode gerar problemas funcionais, estéticos e psicossociais ao paciente. Diferentes terapêuticas estão disponíveis para o tratamento de TD, entre as quais destacamos a técnica restauradora direta com resina composta (RC). Este estudo tem como objetivo relatar e discutir duas técnicas restauradoras com RC em pacientes odontopediátricos, com dentes anterossuperiores fraturados devido ao TD, tendo, por base, o caso clínico de um paciente com 8 anos, do sexo masculino, diagnosticado com fratura coronária do esmalte e da dentina no incisivo central superior direito e do esmalte, no incisivo lateral superior direito. A restauração dos dentes fraturados foi realizada com sistema adesivo (SA) e RC, sob isolamento relativo do campo operatório. No incisivo central superior direito, devido à extensão da área, foi utilizada a técnica da matriz de silicone para a restauração anatomofuncional da face palatina, confeccionada previamente, a partir do enceramento do modelo estudo. Com menor extensão, a restauração do incisivo lateral superior direito foi realizada pela técnica da mão livre. Nesse caso clínico, dentina e esmalte vestibular foram restaurados por meio da estratificação de incrementos de RC com diferentes opacidades. Em fase de proservação clínica, os resultados estético e funcional obtidos foram considerados altamente satisfatórios para os pacientes, familiares e operadores.

DESCRITORES | Restauração Dentária Permanente; Estética Dentária; Resinas Compostas.

ABSTRACT | Direct restorative techniques for fractured anterior teeth in pediatric patient • Dental trauma (DT) can compromise teeth, periodontium, and soft tissues. Fairly common among children and teenagers, DT is a public health problem worldwide, for its high prevalence on upper central incisors can trigger functional, aesthetic, and psychosocial issues in patients. Several approaches are possible for properly treating DT, such as the direct restoration using resin-based composite (RC). This study aimed to report and discuss two restorative techniques using RC in an 8-years old male child with upper anterior teeth fractured due to DT, including coronal fracture involving enamel and dentin in the right maxillary central incisor, and only enamel in the right maxillary lateral incisor. The restoration was performed using adhesive system (AS) and RC under relative isolation of the operatory field. Due to the large fractured area in the right maxillary central incisor, a silicon guide was previously prepared based on a waxing model to facilitate the reestablishment of the lingual surface form and anatomy. Having had a smaller fractured area, the right maxillary lateral incisor was restored using the direct free-hand technique. In this clinical case, enamel and detin were restored by incremental filling technique with different resin opacities. In the follow-up clinical evaluation, the patient, relatives, and dental clinicians reported satisfactory functional and aesthetic outcomes.

DESCRIPTORES | Permanent Dental Restoration; Dental Aesthetics; Composite Resins.

AUTOR CORRESPONDENTE

\footnotetext{
- Danielle Liêda Cunha Fróes Clínica Odontológica Universitária, Universidade Estadual de Londrina (UEL) • Rodovia Celso Garcia Cid, PR 445, Km 380, $\mathbf{s} / \mathbf{n}$ Londrina, PR, Brasil • 86057-970 E-mail: liedafroes@gmail.com

- Recebido April 05, 2021 • Aceito May 08, 2021

- Dol http://dx.doi.org/10.11606/issn.2357-8041.clrd.2019.185355
} 


\section{INTRODUÇÃO}

Frequente na infância e adolescência, o traumatismo dentário (TD) é considerado um problema de saúde pública, e os impactos estético, psicossocial e monetário por ele causados podem comprometer a qualidade de vida do paciente e dos seus familiares. Na dentição permanente, fatores como sobressaliência acentuada e proteção labial inadequada aumentam a susceptibilidade dos dentes anterossuperiores à fratura por $\mathrm{TD}$, sendo a fratura coronária, sem comprometimento do tecido pulpar, o dano que ocorre com maior frequência. ${ }^{1}$

O prognóstico do tratamento proposto para dentes traumatizados depende do diagnóstico detalhado dos tecidos dentários comprometidos pelo trauma, da qualidade e quantidade da estrutura dentária remanescente, da disponibilidade do fragmento dentário em condições para ser utilizado, do tempo decorrido entre o acidente e o primeiro atendimento, da necessidade estética do paciente e de seus responsáveis, da base científica e da habilidade do cirurgião-dentista para planejar e executar o procedimento. ${ }^{2}$

Em dentes permanentes e diante da inviabilidade da colagem do fragmento dentário, a restauração direta com resina composta (RC), quando realizada com rigor técnico e científico, permite o restabelecimento estético e funcional do dente fraturado, somado à inclusão social do paciente, com menor custo e tempo clínico em relação às restaurações estéticas indiretas.

Fatores como extensão da fratura coronária, habilidade do profissional, necessidade estética do paciente/responsável, colaboração do paciente e tempo clínico disponível para a execução do procedimento devem ser considerados na seleção da técnica restauradora com $\mathrm{RC},{ }^{3}$ sendo as mais praticadas a técnica da: 1) mão livre, com a utilização somente de espátulas de inserção de RC e pincéis; e 2) utilização de guia ou muralha para restauração da face lingual (ou palatina), a partir do posicionamento e estabilização de tira matriz de poliéster ou a partir da confecção de matriz com silicone de condensação ou adição, após moldagem, obtenção de modelo de gesso e enceramento da área a ser restaurada.

Portanto, por meio do relato de um caso clínico, o objetivo deste trabalho é apresentar e discutir a técnica de restauração direta da estética, anatomia e função dos dentes anteriores permanentes com fratura coronária, devido ao TD, em paciente odontopediátrico.

\section{RELATO DE CASO}

Um paciente do sexo masculino, com 8 anos de idade, compareceu ao Pronto Socorro Odontológico da Clínica Odontológica Universitária da Universidade Estadual de Londrina (PSO-COU/UEL) encaminhado pelo Pronto Atendimento Infantil de Londrina.

$\mathrm{Na}$ anamnese, quando questionada, a mãe, responsável legal do paciente, relatou que o mesmo: "caiu, brincando em casa, bateu o dente e está doendo". No exame físico intrabucal, foi diagnosticada fratura coronária do esmalte e da dentina, no incisivo central superior direito, e do esmalte (bordo incisal), no incisivo lateral superior direito (Figura 1). A resposta do paciente ao teste de percussão vertical e horizontal não foi sugestiva a alterações endodôntica e periodontais, respectivamente. O comprometimento da inserção periodontal também foi descartado por meio do teste de mobilidade no sentido vestíbulopalatino. O exame radiográfico (Figura 2) ratificou o não comprometimento periodontal e periapical, bem como a não fratura radicular. A conduta de urgência foi, na sequência: limpeza da dentina exposta do incisivo central superior direito com clorexidina 0,12\% (Perioplak, Reymer, Aparecida de Goiânia-GO, Brasil), aplicada com auxílio de bolinha de algodão estéril, e proteção pulpar indireta, com cimento de ionômero de vidro (CIV) convencional (Maxxion R, FGM, Joinville$\mathrm{SC}$, Brasil), devido à sintomatologia dolorosa (Figura 3). Não houve necessidade de prescrição medicamentosa, pois o paciente relatou alívio da sintomatologia 
após a inserção do CIV. Ao término, o paciente foi encaminhado para o programa de Residência em Odontologia área de Dentística da UEL.

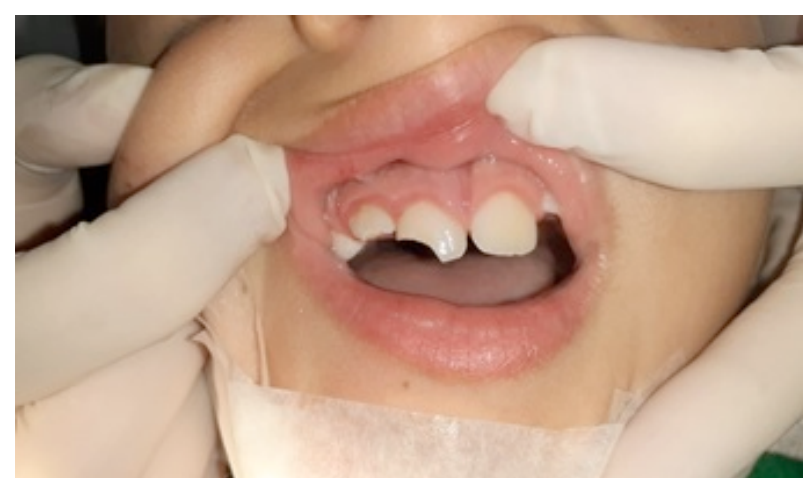

FIGURA 1 | Condição clínica inicial mediata após traumatismo dentário.

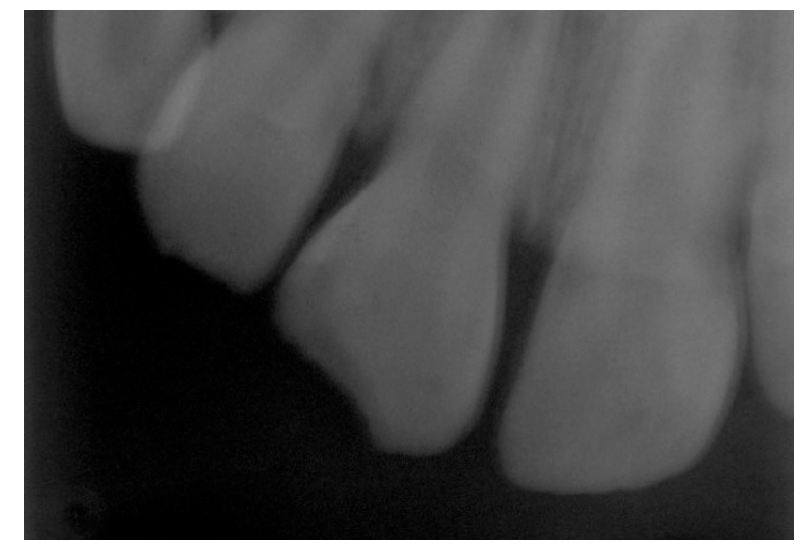

FIGURA 2 | Radiografia periapical região do incisivo central superior direito (11) e incisivo lateral superior direito (12).

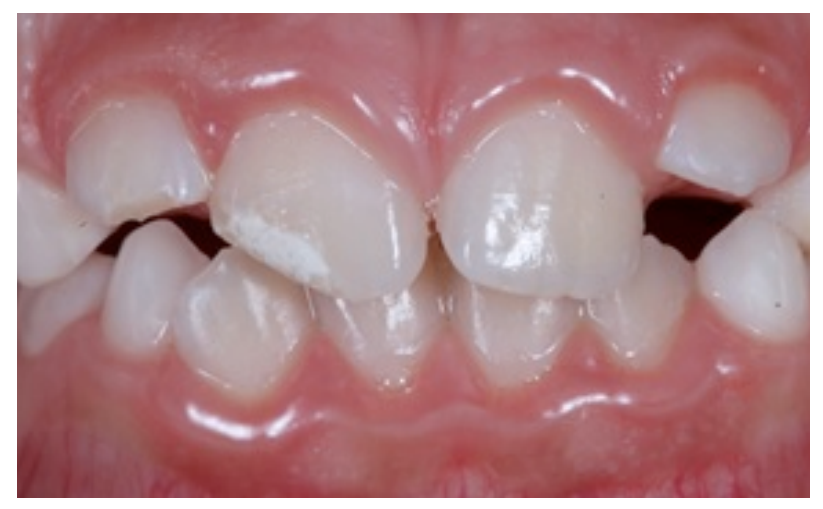

FIGURA 3 | Condição clínica imediata após atendimento de urgência.
Passados 11 dias, o paciente foi atendido para reavaliação. Na consulta, foi realizada moldagem da arcada superior com alginato (Jeltrate Dustless, Dentsply, Pirassununga-SP, Brasil) para obtenção de modelo de estudo e enceramento do incisivo central superior direito.

Na sessão seguinte, 26 dias após o atendimento emergencial no PSO-COU/UEL, o paciente foi atendido para restauração direta em RC do incisivo central superior direito. Considerando a extensão da fratura, foi utilizada guia para restauração da face palatina, obtida a partir da confecção de matriz de silicone (Perfil, Coltene, Rio de Janeiro-RJ, Brasil).

Por razões didáticas, os materiais odontológicos utilizados estão apresentados de acordo com a sequência clínica: 1) Anestesia infiltrativa, com mepivacaína 2\% (vasoconstritor adrenalina 1:100.00o), para remoção do CIV, com broca esférica \#2 Jet carbide (Labordental, São Paulo-SP, Brasil), montada em baixa rotação; 2) Acabamento do esmalte no ângulo cavo superficial, com ponta diamantada \#119oF (Kavo, Joinville-SC, Brasil) (Figura 4); 3) Profilaxia dos dentes anterossuperiores com pasta de pedra-pomes e água, com auxílio de escova de Robson (Ultra-soft, American Burrs, Palhoça-SC, Brasil), montada em baixa rotação; 4) Seleção da cor da RC a ser utilizada a partir da verificação da matiz e croma dos dentes a serem restaurados com auxílio da escala Vita Clássica (Vita Lumin, Vita Zahnfabrik, Bad Sackingen, Alemanha). A partir de então, sob isolamento relativo do campo operatório e sem a iluminação do refletor, foi realizada restauração de diagnóstico para ratificação das cores $\mathrm{A} 1 \mathrm{E}$ (Z35o XT Filtek, 3M, Sumaré-SP, BR) para esmalte e A1B (Z35O XT Filtek, 3M, Sumaré-SP, Brasil) para dentina. Valendo ressaltar que a espessura dos incrementos para essa restauração foi semelhante à espessura da restauração final desejada; 5) Isolamento relativo dos dentes anterossuperiores com roletes de algodão (Cremer, Blumenau-SC, Brasil) e afastador labial 
(Expandex Infantil, Indusbello, Londrina-PR, Brasil); 6) Condicionamento ácido do esmalte e da dentina com ácido fosfórico $37 \%$ (Biodinâmica, Ibiporã-PR, Brasil), durante 30 segundos em esmalte e 15 segundos em dentina; 7) Lavagem com jato de água para remoção do agente condicionador durante 30 segundos; 8) Troca dos roletes de algodão; 9) Secagem do campo operatório com jato de ar e da dentina condicionada com filtro de papel (Melitta, São Paulo-SP, Brasil; 10) Aplicação de duas camadas do sistema adesivo (SA) (Adper Single Bond 2, 3M ESPE, Sumaré-SP, Brasil), com auxílio de pincel microaplicador (Cavibrush regular, FGM, Joinville-SC, Brasil), de forma ativa, por 15 segundos (Figura 5); 11) Remoção do excesso de SA com auxílio de pincel microaplicador limpo e intervalo de tempo para evaporação do solvente; 12) Fotoativação do SA com aparelho à base de diodo emissor de luz (LED) (Emitter B Schuster, Santa Maria-RS, Brasil), com potência de $1250 \mathrm{mw} / \mathrm{cm}^{2}$, por 20 segundos; 13) Posicionamento da matriz de silicone (Figura 6) para restauração da face palatina a partir da inserção da RC de esmalte, cor A1E (Z350 XT Filtek, 3M, SumaréSP, Brasil), e fotoativação da polimerização com o aparelho à base de LED; 14) Na sequência, por meio da técnica incremental, com auxílio de espátula de inserção, pincel e sonda exploradora, foi realizada a restauração da área correspondente à dentina com a RC de cor A1B (Z350 XT Filtek, 3M, Sumaré-SP, Brasil), com caracterização do volume e anatomia (sulcos de desenvolvimento) desse substrato. Por apresentar maior grau de opacidade que a RC de esmalte, a RC A1B também foi inserida sobre o ângulo cavo superficial para mascarar a linha de transição entre dente e área restaurada. Por último, a face vestibular foi restaurada por meio da inserção da RC cor A1E (Z350 XT Filtek, 3M, Sumaré-SP, Brasil). Todos os incrementos de RC foram fotopolimerizados por 40 segundos, com a unidade fotoativadora à base de LED já descrita; 15)
Acabamento imediato da face proximal com lâmina de bisturi $\mathrm{n}^{\mathrm{o}} 12$ (Feather Safety Razor, Osaka, Japão) da face palatina com broca multilaminada \#9406 (Microdont, São Paulo-SP, Brasil) e da face vestibular com discos de lixa (Diamond Master, FGM Produtos Odontológicos, Joinville-SC, Brasil); e 16) 7 dias após, foi realizada a caracterização (texturização) da face vestibular com ponta diamantada \#3195F (Fava, Parque Paulista Franco da Rocha-SP, Brasil) e polimento final, com borracha abrasiva em formato de taça, impregnada com sílica (Jiffy®), Ultradent, Indaiatuba-SP, Brasil) e disco de feltro Diamond Flex (FGM, Joinville-SC, Brasil) em associação à pasta de polimento à base de óxido de alumínio Diamond $\mathrm{R}$ (FGM, Joinville-SC, Brasil) (Figura 7).

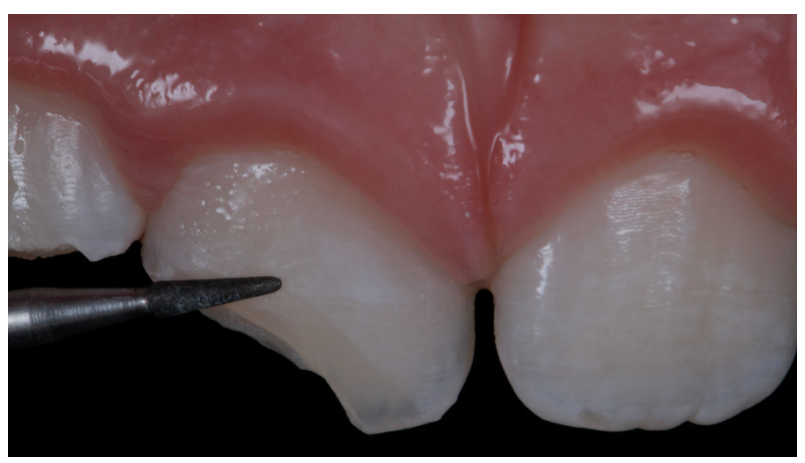

FIGURA 4 | Acabamento do esmalte no ângulo cavo superfical vestibular.

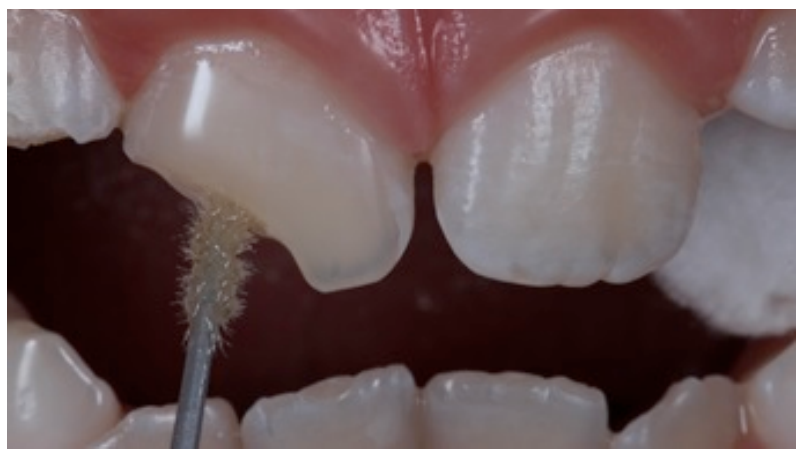

FIGURA 5 | Aplicação do sistema adesivo nos substratos condicionados. 


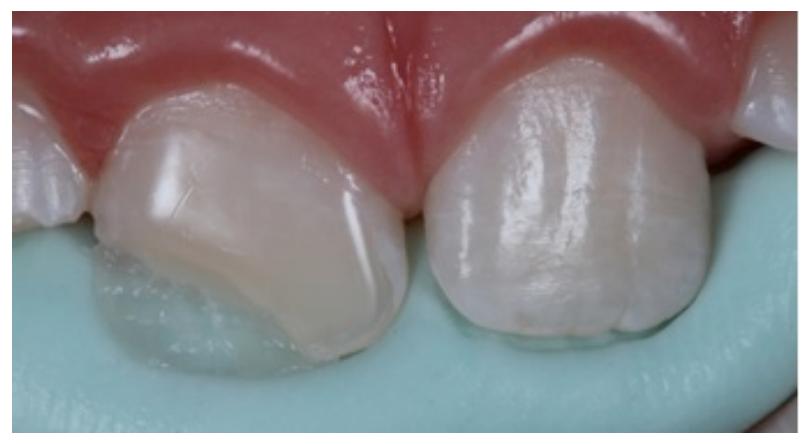

FIGURA 6 | Matriz de silicone em posição para auxiliar na restauração da face palatina.

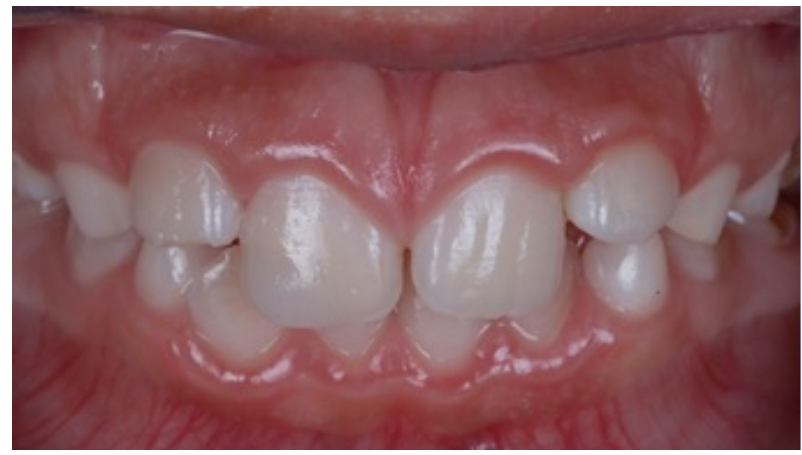

FIGURA 7 Restauração do dente 11 após acabamento e polimento final.

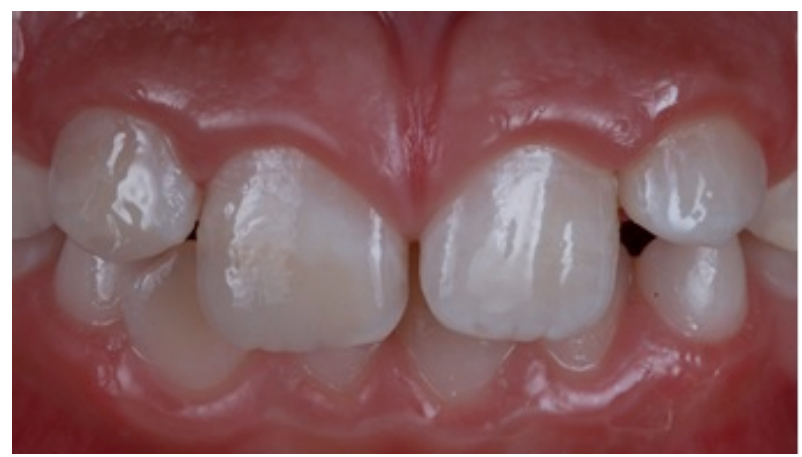

FIGURA 8 | Restauração do dente 12 após acabamento e polimento final.

Após uma semana, o paciente foi atendido para restauração direta em $\mathrm{RC}$ do incisivo lateral superior direito. Os materiais odontológicos foram utilizados, basicamente, na mesma sequência clínica descrita para a restauração do incisivo central superior direito, entretanto, considerando a pouca extensão da fratura (esmalte), a restauração foi pela técnica da mão livre e foi selecionada apenas uma RC de esmalte na cor A1E (Z350 XT Filtek, 3M, Sumaré-SP, Brasil) (Figura 8).

\section{DISCUSSÃO}

O presente trabalho fornece informações técnicas fundamentadas na ciência e relevantes para otimizar o diagnóstico e a tomada de decisão no tratamento de TD, o que justifica sua apresentação. O resultado clínico obtido evidencia que uma abordagem conservadora, desde que possível, é fundamental à manutenção da dentição natural e das estruturas periodontais. Sendo o TD mais prevalente em crianças do sexo masculino e com acometimento nos incisivos centrais superiores, ${ }^{4}$ os dados do caso clínico relatado estão em consonância com a literatura.

A restauração de dentes anteriores com fratura coronária, decorrente do TD, pode ser realizada por meio da colagem autógena ou homógena do fragmento coronário, ou por meio de restauração direta com RC, pela técnica da mão livre, também sendo possível a utilização de guia para restauração da face lingual. Assim, considerando a ausência do fragmento dentário para a colagem autógena e o estágio atual das RC, as restaurações diretas são uma opção conservadora e aceitável à obtenção de resultados estéticos e funcionais satisfatórios.

A restauração de dentes anteriores com $\mathrm{RC}$ pela técnica da mão livre exige do profissional conhecimento teórico quanto às características anatômicas do dente a ser restaurado e a habilidade para inserção e escultura do material. Assim, considerando o tempo clínico necessário à realização das restaurações, a extensão da área a ser restaurada, a maior previsibilidade dos resultados desejados, a idade e a condições emocionais do paciente, optouse, neste caso, pela utilização da técnica com guia para restauração da face palatina, exceto no incisivo lateral superior direito, por envolver somente o esmalte. Essa técnica torna mais fácil a execução da 
restauração e exige menor tempo para o acabamento e polimento da restauração. ${ }^{5}$

Considerando o atendimento ser de um paciente pediátrico não condicionado ao uso do isolamento absoluto, realizou-se o isolamento relativo, visando minimizar o tempo clínico e a possibilidade de estresse do paciente. Para tal, as restaurações dos dentes foram realizadas em duas sessões clínicas. Durante todas as sessões restauradoras, para controle da umidade relativa local, além de roletes de algodão, também foram utilizados afastador labial, abridor de boca, sucção constante de alta potência e trabalho à quatro mãos.

Para melhor previsibilidade dos resultados desejados, foi realizada a restauração diagnóstica. Essa etapa clínica corresponde à inserção e fotoativação da RC, sem condicionamento e aplicação do SA, para definir, de maneira assertiva, a cor e a espessura da restauração a ser confeccionada. Considerando a idade e o baixo nível de exigência estética do paciente e pais/responsáveis legais, o ensaio restaurador foi realizado na mesma sessão clínica de realização da restauração definitiva. Para situações clínicas de maior rigor e necessidade estética, é recomendado intervalo entre a sessão clínica de realização da restauração diagnóstica e a confecção da restauração definitiva.

Previamente às restaurações, o preparo dos dentes consistiu apenas no acabamento do ângulo cavo superficial para a remoção de esmalte friável remanescente, sem nenhum desgaste adicional.

No caso clínico descrito, o SA utilizado foi o convencional de dois passos (Adper Single Bond 2, $3 \mathrm{M}$ ), que apresenta problemas relatados quanto à toxicidade do componente BIS-GMA ${ }^{6}$ e a coloração amarelada conferida pela canforoquinona, que pode causar alteração na cor da restauração. ${ }^{7}$ Apesar dessas desvantagens, o resultado mediato foi satisfatório, sendo o conhecimento e a habilidade do operador considerados mais relevantes que a composição do material.

Nesse contexto, tecnicamente é importante considerar que a espessura do SA é um fator fundamental na longevidade da restauração, por isso, é de extrema importância a remoção do excesso de SA aplicado. ${ }^{8}$ No caso relatado, o excesso de SA foi removido com auxílio de pincel microaplicador limpo, como forma de minimizar os efeitos adversos referentes à qualidade do ar.

Após a confecção da face palatina da restauração, os espaços correspondentes à dentina e ao esmalte foram restaurados pela técnica incremental. Nela, os incrementos simulam os tecidos dentários para que a interação do compósito com a luz seja semelhante à dos dentes naturais. Além disso, a inserção de incrementos de até $2 \mathrm{~mm}$ de espessura permite melhor fotoativação, reduz o stress da contração de polimerização e confere boa integridade marginal. ${ }^{9}$

As restaurações somente foram consideradas concluídas após a realização das etapas de acabamento, imediato e mediato, e polimento, mediato. A realização de polimento mediato de restaurações de $\mathrm{RC}$ reduz a formação de fendas e microtrincas, consequentemente, reduz a probabilidade de manchamento e favorece a melhor adaptação marginal. ${ }^{10}$

\section{CONCLUSÃO}

Restaurações diretas em RC são opções viáveis e conservadoras no reestabelecimento anatomofuncional dos elementos acometidos por TD em pacientes odontopediátricos. No entanto, para alcançar o sucesso clínico, o acompanhamento clínico e radiográfico a longo prazo é fundamental, permitindo monitorar o desempenho da restauração e possíveis complicações tardias ao trauma dentário, como as alterações pulpares e de coloração do substrato. 


\section{REFERÊNCIAS}

1. Bastone EB, Freer TJ, Mcnamara JR. Epidemiology of dental trauma: a review of the literature. Aust Dent J. 2000; 45(1):2-9. Doi: https://doi.org/10.1111/j.1834-7819.200o. tboo234.x

2. Kina M, Ribeiro LG, Monteiro S Jr, Andrada MA. Fragment bonding of fractured anterior teeth: case report. Quintessence Int. 2010;41(6):459-61.

3. Nahsan FPS, Mondelli RFL, Franco EB, Naufel FS, Ueda JK, Schmitt VL, et al. Clinical strategies for esthetic excellence in anterior tooth restorations: understanding color and composite resin selection. J Appl Oral Sci. 2012;20(2):151-6. Doi: https://doi.org/10.1590/S1678-77572012000200005

4. Traebert J, Almeida ICS, Garghetti C, Marcenes W. Prevalência, necessidade de tratamento e fatores predisponentes do traumatismo na dentição permanente de escolares de 11 a 13 anos de idade. Cad Saude Publica. 2004;20(2):403-10. Doi: https://doi.org/10.1590/So102-311X2004000200007

5. Mishra A, Yeluri R, Garg N, Rallan M. Putty silicone as a guide in the restorative management of primary double tooth: a case report. Ann Dent Spec. 2015;3(1):21-3.

6. Kuan YH, Huang FM, Lee SS, Li YC, Chang YC. Bisgma stimulates prostaglandin E2 production in macrophages via cy- clooxygenase-2, cytosolic phospholipase A2, and mitogen-activated protein kinases family. PLoS One. 2013;8(12):e82942. Doi: https://doi.org/10.1371/journal.pone.0082942

7. Oliveira OF Jr, Kunz PVM, Baratto Filho F, Correr GM, Cunha LF, Gonzaga CC. Influence of pre-curing different adhesives on the color stability of cemented thin ceramic veneers. Braz Dent J. 2019;30(3):259-65. Doi: http://doi.org/10.1590/01036440201902369

8. Ausiello P, Apicella A, Davidson CL. Effect of adhesive layer properties on stress distribution in composite restorations-a 3D finite element analysis. Dent Mater. 2002;18(4):295-303. Doi: https://doi.org/10.1016/So109-5641(01)00042-2

9. Betrisey E, Krejci I, Di Bella E, Ardu S. The influence of stratification on color and appearance of resin composites. Odontology. 2016;104(2):176-83. Doi: https://doi.org/10.1007/ s10266-015-0197-2

10. Pettini F, Corsalini M, Savino MG, Stefanachi G, Di Venere D, Pappalettere C, et al. Roughness analysis on composite materials (microfilled, nanofilled and silorane) after different finishing and polishing procedures. Open Dent J. 2015;9:35767. Doi: https://doi.org/10.2174/1874210601509010357 